\title{
Numerical Resolution of Emden's Equation using Adomian Polynomials
}

\author{
Paper Type: Research paper \\ Keywords: Emden's equation, Power series, Frobenius method, Adomian polynomials, Decomposition
} Method.

\section{Purpose of the paper:}

In this paper we show the advantages of using the decomposition method introduced by Adomian to solve Emden's equation, a classical non-linear equation that appears in the study of the thermal behaviour of a spherical cloud and of the gravitational potential of a polytropic fluid at hydrostatic equilibrium.

\section{Design/methodology/approach:}

In our work, we first review Emden's equation and its possible solutions using the Frobenius and Power series methods; then, Adomian polynomials are introduced. Afterwards, Emden's equation is solved using Adomian's decomposition method and, finally, we conclude with a comparison of the solution given by Adomian's method with the solution obtained by the other methods, for certain cases where the exact solution is known.

\section{Findings:}

Solving Emden's equation for $n$ in the interval [0, 5] is very interesting for several scientific applications, such as astronomy. However, the exact solution is known only for $\mathrm{n}=0, \mathrm{n}=1$ and $\mathrm{n}=5$. The experiments show that Adomian's method achieves an approximate solution which overlaps with the exact solution when $n=0$, and that coincides with the Taylor expansion of the exact solutions for $n=1$ and $n=5$. As a result, we obtained quite satisfactory results from our proposal.

\section{Originality/value of the paper:}

The main classical methods for obtaining approximate solutions of Emden's equation have serious computational drawbacks. We make a new, efficient numerical implementation for solving this equation, constructing iteratively the Adomian polynomials, which leads to a solution of Emden's equation that 
extends the range of variation of parameter $n$ compared to the solutions given by both the Frobenius and the power series methods.

\section{INTRODUCTION}

Emden's equation is a classical non-linear equation that appears in the study of the thermal behaviour of a spherical cloud of gas acting under the mutual attraction of its molecules and subject to the classical laws of thermodynamics (Chandrasekar, 1958; Nagle et al., 2011). In astrophysics, it also expresses the gravitational potential of a self-gravitating, spherically symmetric and polytropic fluid at hydrostatic equilibrium. It is represented by the equation:

$$
y^{\prime \prime}+\frac{2}{x} y^{\prime}+y^{n}=0
$$

associated with the initial conditions $y(0)=1, \quad y^{\prime}(0)=0, \quad n \in Z^{+}$.

When $n=1$, the equation is called Lane-Emden's first-order equation. The problem posed by Emden, in early 1900s, can be stated in the following way: "Determine the first point on the positive $X$-axis where the solution to the initial value problem

$$
x y^{\prime \prime}+2 y^{\prime}+x y=0
$$

with $y(0)=1, \quad y^{\prime}(0)=0$, is zero". The solution to Lane-Emden's equation is

$$
y(x)=\frac{\sin x}{x}
$$

The first zero of the solution corresponds to the first zero of the numerator, that is, $x=\pi$.

This equation is used by astronomers and astrophysicists to calculate the internal temperature and density of certain stars and nebulae. It was used to model several phenomena in mathematical Physics and astrophysics such as the theory of stellar structure, the thermal behaviour of a spherical cloud of gas, isothermal gas spheres and theory of thermionic currents (Hasan and Zhu, 2007).

Many different numerical solutions for this equation have been proposed in the last few years. To cite only a few, in (Karimi Vanani and Aminataei, 2010) an approximate polynomial solution is presented for solving Lane-Emden equations as singular initial value problems; (Yiğider et al., 2011) proposed a Padé approximation method for solving differential equations of Lane-Emden type with effective results; (He, 
2003) used the semi-inverse method for obtaining a variation of the Lane-Emden equation, and obtained good results when applying finite element methods or Ritz method; (Harley and Momoniat, 2010) proposed an efficient method for determining an initial guess for an iterative solution to a boundary value problems for Lane-Emden type equations; finally, in (Aslanov, 2009) an extension of Adomian's decomposition method for different types of Emden-Fowler-like equations is introduced.

In this work, we shall solve Lane-Emden's equation using Adomian polynomials, for the interval $n \in[0,5]$, comparing the results to exact solutions, which are known for $n=0,1$ and 5 . This paper is organized as follows: the solution of Lane-Emden's equation using the Frobenius and power series methods is shown in Section 2. Then, Section 3 focuses on the decomposition method presented by Adomian, giving its key concepts and main applications. Afterwards, Section 4 describes how to solve Lane-Emden's equation using Adomian polynomials and compares the achieved solution with the exact ones. Finally, we shall conclude with some remarks of our research.

\section{SOLUTION USING THE FROBENIUS AND POWER SERIES METHODS}

Lane-Emden's equation has two major difficulties in order to be solved. First of all, the equation has variable coefficients, and secondly, the equation has a regular-singular point at $x=0$.

The general solution of the Lane-Emden's equation:

$$
y^{\prime \prime}+\frac{2}{x} y^{\prime}+y=0
$$

is given by the linear combination of two solutions that are linearly independent, and it can be expressed, after applying the Frobenius method (Nagle et al., 2011), as:

$$
y=C_{1} \frac{\sin x}{x}+C_{2} \frac{\cos x}{x}
$$

where $C_{1}, C_{2}$ are arbitrary constants. The solution $y(x)=\frac{\sin x}{x}$ (see (Chandrasekar, 1958)) satisfies the initial conditions. When $x=\pi$, the Lane-Emden's problem is solved.

For $n=2,3,4, \ldots$, the general solution for Equation (1) can be obtained by using the power series method (i.e., Taylor). The first terms are given by: 


$$
y=1-\frac{x^{2}}{3 !}+n \frac{x^{4}}{5 !}-\ldots
$$

\section{THE DECOMPOSITION METHOD}

Adomian developed a decomposition technique for solving non-linear functional equations (see (Adomian, 1983; Adomian, 1986; Adomian, 1994; Bellman and Adomian, 1985)). It is convenient to write then in the form:

$$
u-N(u)=f
$$

where $N$ is a non-linear operator from a Hilbert space $H$ into $H$ and $f$ is a given function in $H$. Let us assume that Eq. (7) has a unique solution. Adomian's technique proposes to obtain the solution to Eq. (7) as an infinite series:

$$
u=\sum_{i=0}^{\infty} u_{i}
$$

as well as to expand the non-linear term $N(u)$ as follows:

$$
N(u)=\sum_{i=0}^{\infty} A_{i}
$$

where $A_{i}$ are polynomials that depend uniquely on $u_{0}, u_{l}, \ldots, u_{i}$, and are known as Adomian polynomials.

Replacing Eqs. (8) and (9) in Eq.(7), we obtain:

$$
\sum_{n=0}^{\infty} u_{n}-\sum_{n=0}^{\infty} A_{n}=f
$$

The terms of the series $\sum_{n=0}^{\infty} u_{n}$ are obtained by identification in Eq. (10):

$$
\left\{\begin{array}{l}
u_{0}=f \\
u_{1}=A_{0} \\
\vdots \\
u_{n+1}=A_{n}
\end{array}\right.
$$

where $A_{k}\left(u_{0}, \ldots, u_{k}\right)$ are certain polynomials of $u_{0}, \ldots, u_{k}$. The series exact solution of Eq. (7) is thus achieved. 
On the other hand, $A_{i}$ are obtained from the relations:

$$
v=\sum_{i=0}^{\infty} \lambda^{i} u_{i}, \quad N\left(\sum_{i=0}^{\infty} \lambda^{i} u_{i}\right)=\sum_{i=0}^{\infty} \lambda^{i} A_{i}
$$

where $\lambda$ is a parameter. The $A_{n}$ can be obtained formally (see (Adomian, 1983; Adomian, 1986; Adomian, 1994)) from Eq. (12) by the expression:

$$
n ! A_{n}=\frac{d^{n}}{d \lambda^{n}}\left[N\left(\sum_{i=0}^{n} \lambda^{i} u_{i}\right)\right]_{\lambda=0}
$$

The definition of the polynomials $A_{j}$ in Eq. (13) shows that $A_{j}$ only depend on $u_{0}, u_{l}, \ldots, u_{j}$ and not on $u_{j+l}$, $u_{j+2}, \ldots$ For real functions $N$, we have that $A_{n}$ can be found by (Abbaoui, 1995):

$$
\left\{\begin{array}{l}
A_{0}\left(u_{0}\right)=N\left(u_{0}\right) \\
A_{n}\left(u_{0}, \ldots, u_{n}\right)=\sum_{\alpha_{1}+\ldots+\alpha_{n}=n} N^{\left(\alpha_{1}\right)}\left(u_{0}\right) \frac{u_{1}^{\left(\alpha_{1}-\alpha_{2}\right)}}{\left(\alpha_{1}-\alpha_{2}\right) !} \ldots \frac{u_{n-1}^{\left(\alpha_{n-1}-\alpha_{n}\right)}}{\left(\alpha_{n-1}-\alpha_{n}\right) !} \cdot \frac{u_{n}^{\alpha_{n}}}{\alpha_{n} !}, \quad \text { for } n \neq 0
\end{array}\right.
$$

where the succession $\left(\alpha_{i}\right)_{i=1, . ., n}$ is decreasing.

Proofs of convergence are given in (Abbaoui, 1995; Abbaoui et al., 2001; Chandrasekar, 1958; Cherruault and Adomian, 1993; Cherruault et al., 1995). In (Abbaoui, 1995) it is stated that if the nonlinear operator satisfy the condition $\|N(u)\| \leq M \quad \forall n \in N, \quad M<\frac{1}{e}$ then $\left\|u_{n+1}\right\|=\left\|A_{n+1}\right\| \leq M^{n+1} e^{n+1}$. Therefore, if we denote as $e_{k}$ the difference between the exact solution $u$ (sum of the series) and $\varphi_{k}$ (sum of the truncated series in $k$ terms) we have:

$$
\left\|e_{k}\right\| \leq\left\|u-\varphi_{k}\right\|=\left\|\sum_{i=k}^{\infty} u_{i}\right\| \leq \sum_{i=k}^{\infty}(M e)^{i}=\frac{M^{k} e^{k}}{1-M e}
$$

Therefore, since $M e<1$, then $\lim _{k \rightarrow \infty}\left\|e_{k}\right\|=0$. A convergence theorem was published in (Abbaoui et al., 2001), with weaker hypotheses than the above, and therefore applicable to a wider range of equations. The calculus of the Adomian polynomials are given in (Abbaoui, 1995). Computational implementations for applications of Adomian polynomials to different fields have been carried out in recent years, such as Population dynamics (Bordehore et al., 2003), Diffusion problems (Pujol and Grimalt, 2003), Meteorology (Guellal et al., 1997), Hydrology (Guellal et al., 2000), Chemistry kinetics (Grimalt and Pujol, 1999), Physics (Fowler, 1931), the Fokker-Planck equation (Tatari et al., 2007), Reaction 
mechanisms and convection effects (Hashim et al., 2006) and Combustion problems (Wazwaz, 2005), among others.

\section{EMDEN'S EQUATION SOLVED BY THE DECOMPOSITION METHOD}

Before applying Adomian's method to Equation (1), since through a suitable transformation we will simplify the problem (see (Bellman, 1953), pp. 143-163), let us carry out a change of variables,

$$
v=x, \quad u=v y
$$

then Equation (1) is transformed into

$$
\frac{d^{2} u}{d v^{2}}=-\frac{u^{n}}{v^{n-1}}
$$

with the initial conditions $u(0)=0, \frac{d u}{d v}(0)=1$.

Rewriting Equation (17) in canonical form, we have:

$$
L_{v v} u=-\frac{u^{n}}{v^{n-1}}
$$

where $L_{v v}=\frac{d^{2}}{d v^{2}}$. Let $L_{v v}^{-1}$ denote the double integration with respect to $v$, then Equation (18) can be written as:

$$
u=v-L_{v v}^{-1} u^{n} v^{1-n}
$$

Applying Adomian's method and decomposing $u=\sum_{i=0}^{\infty} u_{i}$ and the non-linear term $N(u)=u^{n}=\sum_{i=0}^{\infty} A_{i}$ in a series (where $A_{i}$ are the Adomian polynomials calculated by the formulae given in Eqs. (13) and (14)), we have:

$$
\sum_{i=0}^{\infty} u_{i}=v-L_{v v}^{-1} \sum_{i=0}^{\infty} A_{i} v^{1-n}
$$

The Adomian scheme is, therefore:

$$
\begin{aligned}
& u_{0}=v \\
& u_{k}=-L_{v v}^{-1} A_{k-1} v^{1-n}, \quad k=1,2, \ldots
\end{aligned}
$$


and, specifically,

$$
\begin{aligned}
& u_{0}=v \\
& A_{0}=N\left(u_{0}\right)=v^{n} \\
& u_{1}=-\int\left(\int A_{0} v^{1-n} d v\right) d v=-\int\left(\int v^{n} v^{1-n} d v\right) d v=-\frac{v^{3}}{6}, \\
& A_{1}=u_{1} N^{\prime}\left(u_{0}\right)=-\frac{n}{6} v^{n+2} \\
& u_{2}=-\int\left(\int A_{1} v^{1-n} d v\right) d v=-\int\left(\int-\frac{n}{6} v^{n+2} v^{1-n} d v\right) d v=n \frac{v^{5}}{5 !}
\end{aligned}
$$

So the solution using Adomian's technique is $u=u_{0}+u_{1}+u_{2}+\cdots=v-\frac{v^{3}}{3 !}+n \frac{v^{5}}{5 !}-\cdots$. Undoing the variable change $y=\frac{u}{v}, \quad v=x$, then we obtain:

$$
\begin{aligned}
& y=1-\frac{1}{3 !} x^{2}+\frac{1}{5 !} n x^{4}-\frac{1}{7 !}\left(\frac{8}{3} n^{2}-\frac{5}{3} n\right) x^{6}+ \\
& +\frac{1}{9 !}\left(\left(\frac{8}{3} n^{3}-\frac{5}{3} n^{2}\right)+7 n^{2}(n-1)+\frac{35}{9} n(n-1)(n-2)\right) x^{8}+\cdots
\end{aligned}
$$

which is analogous to the result obtained by the power series method.

It is particularly interesting to solve Emden's equation for $n \in[0,5]$, a range of great interest in astronomy. The exact solution is known for $n=0, n=1$ and $n=5$, being, respectively:

$$
y=1-\frac{1}{6} x^{2}, \quad y=\frac{\sin x}{x}, \quad y=\left(1+\frac{x^{2}}{3}\right)^{-1 / 2} .
$$

For other values of the parameter analytical forms of the solutions are not known, and they must be obtained numerically.

\section{Results}

There are two main methods for obtaining approximate solutions; see (Burt, 1987; Ditto and Picket, 1990) and, particularly, (Fowler, 1931), which is a classic and very detailed reference. The first of these related 
works transforms Emden's equation into a Riccati integral/differential equation, whereas the second one assumes a power series solution. Both methods lead to a non-perturbative approximate solution, but both have serious computational drawbacks. The first method requires a Riccati equation to be solved for each iteration. However, in the second method, finding the coefficients of the power series by direct substitution is not an easy task, being especially difficult for the higher terms. The Taylor method can be used for both linear and non-linear equations, but has the major drawback when obtaining the derivatives, since these may get too complicated as the order increases. It is not always possible to locate the radius of convergence of the series either, given that there are only existence theorems for linear equations.

Therefore, in order to overcome these problems, we applied Adomian's method to Emden's equation and solved it for any value of the parameter $n$ (notice that $n$ does not necessarily have to be an integer number). Let's write out the Adomian solution for several values of $n$, with seven terms in the series:

$$
\begin{aligned}
& n=0 \quad y=1-\frac{1}{3 !} x^{2} \\
& n=1 \quad y=1-\frac{1}{3 !} x^{2}+\frac{1}{5 !} x^{4}-\frac{1}{7 !} x^{6}+\frac{1}{9 !} x^{8}-\frac{1}{11 !} x^{10}+\frac{1}{13 !} x^{12} \\
& n=2 \quad y=1-\frac{1}{3 !} x^{2}+\frac{1}{60} x^{4}-\frac{11}{7560} x^{6}+\frac{1}{8505} x^{8}-\frac{67}{10692000} x^{10}+\frac{2813}{4378374000} x^{12} \\
& n=3 \quad y=1-\frac{1}{3 !} x^{2}+\frac{1}{40} x^{4}-\frac{19}{7 !} x^{6}+\frac{619}{1088640} x^{8}-\frac{12637}{199584000} x^{10}+\frac{1167973}{93405312000} x^{12} \\
& n=4 \quad y=1-\frac{1}{3 !} x^{2}+\frac{1}{30} x^{4}-\frac{1}{140} x^{6}+\frac{43}{27216} x^{8}-\frac{20201}{74844000} x^{10}+\frac{38411}{486486000} x^{12} \\
& n=5 \quad y=1-\frac{1}{3 !} x^{2}+\frac{1}{4 !} x^{4}-\frac{5}{432} x^{6}+\frac{35}{10368} x^{8}-\frac{533}{684288} x^{10}+\frac{32233}{106748928} x^{12}
\end{aligned}
$$

From these results, we must notice that the Adomian solution for $n=0$ is the exact solution. Moreover, the Adomian solutions for $n=1$ and $n=5$ are exactly the Taylor expansion up to seven terms of the exact solutions $y=\frac{\sin x}{x}$ and $y=\left(1+\frac{x^{2}}{3}\right)^{-1 / 2}$, respectively. In Figures 1,2 and 3 we illustrate both the Adomian and the exact solutions for $n=0,1$ and 5 respectively. For $n=0$, it is obvious that the plots overlap. For $n=1$, the Adomian solution is given using seven terms in the series, and for $n=5$, using 15 terms in the series. 


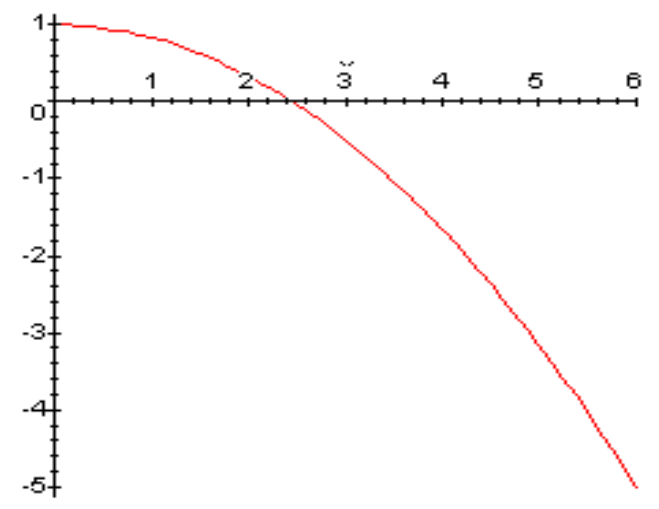

Figure 1: Adomian solution and exact solution for $n=0$.

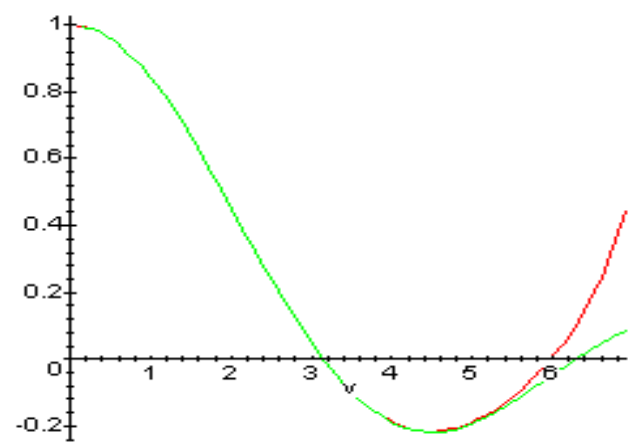

Figure 2: Adomian solution and exact solution for $n=1$.

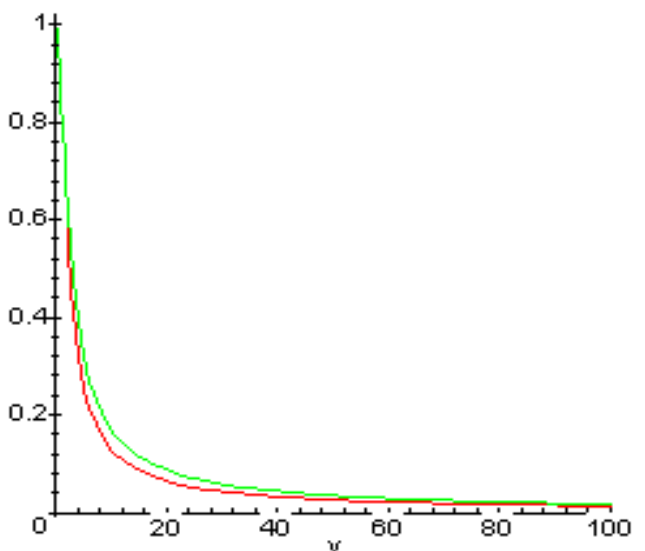

Figure 3: Adomian solution and exact solution for $n=5$.

\section{Conclusions}

In this article we have revised one of the most important equations for modelling polytropic fluids, the Lane-Emden's equation. Thus, the general Emden's equation has been solved using the Adomian method, and for those specific values for which the exact solution is known (namely $n=0,1$ and 5) the Adomian 
solution has been compared to the exact solution, with satisfactory results. The Adomian's method can therefore be seen as a useful tool for solving non-linear functional equations.

\section{ACKNOWLEDGEMENTS}

This work has been supported by the Ministerio de Ciencia e Innovación, project TIN2009-10581.

\section{REFERENCES}

Abbaoui K. (1995), Les fondements mathématiques de la méthode décompositionnelle d'Adomian et application à la résolution des équations issues de la Biologie et de la Médecine, Thèse de Doctorat de l’Université de Paris VI.

Abbaoui K., Pujol M. J., Cherruault Y., Himoun N. and Grimalt P. (2001), "A new formulation of the Adomian method: convergence result”, Kybernetes, Vol. 30 No. 9/10, pp. 1183-1191.

Adomian G. (1983), Stochastic Systems, Academic Press.

Adomian G. (1986), Stochastic Operator Equations, Academic Press.

Adomian G. (1994), Solving Frontier Problems of Physics: The Decomposition Method, Kluwer Academic Publishers.

Aslanov, A. (2009), “Approximate solutions of Emden-Fowler type equations”, International Journal of Computer Mathematics, Vol. 86 No. 5, pp. 807-826.

Bellman R. (1953), Stability Theory of Differential Equations, McGraw-Hill.

Bellman R. and Adomian G. (1985), Partial Differential Equations, Reidel Publishing Company.

Bordehore C., Pascual A., Pujol M. J., Escolano J., Manchón I. and Grimalt P. (2003), "Study and Modelling of a Density Dependent Population Using Faltung (Closed Cycle Type) Equations", International Journal of Numerical Methods for Heat and Fluid Flow, Vol. 13 No. 4, pp. 486-499.

Burt, P. B. (1987), "Nonperturbative solution of nonlinear field equations", Il Nuovo Cimento B, Vol. 100 No. 1, pp. 43-52.

Chandrasekar S. (1958), An introduction to the study of stellar structure, Dover Publications.

Cherruault Y. and Adomian G. (1993), "Decomposition method: A new proof of convergence", Mathematical and Computer Modelling, Vol. 18 No. 12, pp. 103-106. 
Cherruault Y., Adomian G., Abbaoui K. and Rach R. (1995), "Further remarks on convergence of the decomposition method", International Journal of Bio-Medical Computing, Vol. 38 No. 1, pp. 89-93.

Ditto, W. and Pickett, T. (1990), "Exact solutions of nonlinear differential equations using continued fractions", Il Nuovo Cimento B, Vol. 105 No.4, pp. 429-435.

Fowler R. H. (1931), “Further Studies of Emden’s and Similar Differential Equations”, Quarterly Journal of Mathematics, Vol. 45, pp. 289-350.

Grimalt P. and Pujol M. J. (1999), "The decomposition method applied to chemistry kinetics", Información Tecnológica, Vol. 10 No. 3, pp. 129-132.

Guellal S., Cherruault Y., Pujol M. J. and Grimalt P. (2000), "Decomposition method applied to hydrology", Kybernetes, Vol. 29 No. 4, pp. 499-504.

Guellal S., Grimalt P. and Cherruault Y. (1997), “Numerical study of Lorenz's equations by Adomian's method", Computers \& Mathematics with Applications, Vol. 33 No. 3, pp. 25-29.

Harley, C. and Momoniat, E. (2010), "Efficient boundary value problem solution for a Lane-Emden equation", Mathematical and Computational Applications, Vol. 15 No. 4, pp. 613-620.

Hasan, Y. Q. and Zhu, L. M. (2007), "Solving singular initial value problems in the second-order ordinary differential equations", Journal of Applied Sciences, Vol. 7 No. 17, pp. 2505-2508.

Hashim, I., Noorani, M.S.M., Said Al-Hadidi, M.R. (2006), "Solving the generalized Burgers-Huxley equation using the Adomian decomposition method", Mathematical and Computer Modelling, Vol. 43 No. 11-12, pp. 1404-1411.

He, J. H. (2003), "Variational approach to the Lane-Emden equation", Applied Mathematics and Computation, Vol. 143 No. 23, pp. 539-541.

Karimi Vanani, S. and Aminataei, A. (2010), "On the numerical solution of differential equations of Lane-Emden type”, Computers \& Mathematics with Applications, Vol. 59 No. 8, pp. 2815-2820.

Nagle R. K., Saff E. B. and Snider, A. D. (2011), Fundamentals of Differential Equations, Addison Wesley.

Pujol M. J. and Grimalt P. (2003), “A non-linear model of cerebral diffusion: stability of finite differences method and resolution using the Adomian method", International Journal of Numerical Methods for Heat 
and Fluid Flow, Vol. 13 No. 4, pp. 473-485.

Tatari, M., Dehghan, M. and Razzaghi, M. (2007), “Application of the Adomian decomposition method for the Fokker-Planck equation”, Mathematical and Computer Modelling, Vol. 45 No. 5-6, pp. 639-650.

Wazwaz, A. M. (2005), "Adomian decomposition method for a reliable treatment of the Bratu-type equations", Applied Mathematics and Computation, Vol. 166 No. 3, pp. 652-663.

Yiğider, M., Tabatabaei, K. and Çelik, E. (2011), “The Numerical Method for Solving Differential Equations of Lane-Emden Type by Padé Approximation,” Discrete Dynamics in Nature and Society, vol. 2011, pp. 1-9. 